\title{
EL SISTEMA ELECTORAL EN ALEMANIA A EXAMEN*
}

\author{
YOLANDA FERNÁNDEZ VIVAS \\ Profesora Contratada Doctora de Derecho Constitucional \\ Universidad de Alcalá
}

TRC, núm. 45, 2020, pp. 479-501

ISSN 1139-5583

SUMARIO

I. Rasgos definitorios del sistema electoral alemán II. Regulación del sistema electoral. III. Elementos del sistema electoral. IV. A modo de conclusión.

\section{RASGOS DEFINITORIOS DEL SISTEMA ELECTORAL ALEMÁN}

El sistema electoral alemán es uno de los mejor valorados y admirados del mundo, aunque resulta complejo en su aplicación y en su comprensión, hasta el punto de que a pesar de ser considerado un sistema «ejemplar», los propios ciudadanos alemanes reconocen que ni ellos mismos entienden bien el funcionamiento de su sistema electoral ${ }^{1}$.

A diferencia de lo que ocurre en la mayor parte de los estados constitucionales, en el que los textos constitucionales regulan el sistema electoral de sus órganos legislativos, en Alemania no es así. Este hecho es especialmente llamativo, ya que la Ley Fundamental no regula el sistema electoral, ni siquiera sus reglas básicas, sino que es la ley la encargada de configurarlo, de manera que resulte más

* Este trabajo se enmarca en el Proyecto de Investigación DER2017-84733-R, Partidos políticos: origen, función y revisión de su estatuto constitucional, financiado por el Ministerio de Economía y Competitividad/FEDER.

1 Vidal Prado, C., «El sistema electoral alemán como modelo: ventajas e inconvenientes», en Asamblea. Revista Parlamentaria de la Asamblea de Madrid, 26, 2012, p. 218; VÁzQuez LAPuente, M., JimÉnez SERAL, P., «El nuevo modelo de escaños en el sistema electoral alemán», en Cuadernos Manuel Giménez Abad, 7, 2014, p. 118 . 
sencillo su reforma siempre que sea preciso $^{2}$. Esta decisión fue adoptada por la Comisión parlamentaria constituyente (Parlamentarischer Rat), que, con el fin de no repetir los errores cometidos en la época de Weimar, optó por no incluir el sistema electoral en la Constitución y permitir que fuera el legislador ordinario quien regulase y desarrollara el sistema y procedimiento electoral ${ }^{3}$.

El Bundestag viene eligiéndose invariablemente desde 1949 —aunque con importantes reformas a la hora de aplicarlo- de acuerdo con los principios de una representación proporcional vinculada con la elección personal ${ }^{4}$. Es lo que se denomina un «sistema proporcional personalizado», que combina elementos de los llamados sistemas proporcionales y mayoritarios ${ }^{5}$. Actualmente, el Bundestag está integrado, inicialmente, por 598 diputados, de los cuales 299 se eligen a través de distritos electorales uninominales y los otros 299 restantes son elegidos a través de listas electorales a nivel de cada Land'.

En este sistema electoral combinado cada elector tiene dos votos ${ }^{7}$. El primer voto o Erststimme se utiliza en los distritos electorales uninominales. Con el Erststimme el elector vota únicamente a un candidato, y sale elegido aquél que haya obtenido la mayoría simple de los votos. Por lo tanto, el sistema utilizado para el recuento de votos es el mayoritario. ${ }^{8}$ El segundo voto o Zweitstimme se utiliza para votar a una de las listas electorales que los partidos presentan en cada uno de los Länder y el sistema que se utiliza para el recuento de los votos y la atribución de escaños es el proporcional. ${ }^{9}$ Además, hay que resaltar que es este segundo voto el que realmente determina el reparto de los escaños, porque, como ahora veremos con más detalle, el número total de escaños se distribuye entre los partidos políticos de acuerdo con el número de segundos votos que ha obtenido cada uno, y sólo después, una vez que se han repartido los escaños, se tiene en cuenta ese primer voto para determinar qué candidatos ocupan los escaños ${ }^{10}$.

Es precisamente la combinación de elementos propios de los sistemas proporcionales y de los mayoritarios lo que ha hecho que el sistema alemán se haya

2 Meyer, H., «Demokratische Wahl und Wahlsystem», en Isensee / KirChHhof (Eds.), Handbuch des Staats Rechts, vol. III, $3^{\mathrm{a}}$ ed, C. F. Müller, Heidelberg, 2005, p. 537.

3 WILD, M. Die Gleichbeit der Wabl, Duncker \& Humblot, Berlín, 2003, p. 81.

4 BWahlG, art. 1. Nohlen, D., Sistemas electorales y partidos políticos, Fondo de Cultura Económica,

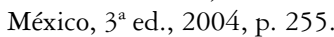

5 Wild, M., Die Gleichbeit der Wabl, p. 217 y ss.; Stern, K., Das Staatsrecht der Bundesrepublik Deutschland, C. H. Beck, Munich, p. 301; Maurer, H., Staatsrecht, $2^{\text {a }}$ ed., C. H. Beck, Munich, 2001, pp. $402-$ 403; Hesse, K., Gründzuge der Verfassungsrechts der Bundesrepublik Deutschland, C.F. Müller, Heidelberg, 1999, p. 254; Badura, P., Staatsrecht, $2^{\text {a }}$ ed., C. H. Beck, Munich, 1996, p. 382.

6 BwahlG, art. 1.

7 BWahlG, art. 4.

8 BWahlG, art. 5.

9 BWahlG, art. 6.

10 G. Leibholz / H.J. Rinck / D. Hesselberger, Grundgesetz, op. cit., Art. 38, Rdn. 236; H.-T. LEE, Chancengleichbeit der politischen Parteien, VVF, Munich, 1994, op. cit., pp. 27-28; SCHMIDT, R., Staatsorganisationsrecht, Schmidt, Grasberg, 2012, p. 37; Mestre-AizPurua, F., «El sistema electoral alemán y su reciente reforma», en Cuadernos Manuel Giménez Abad, 3, 2012, p. 80. 
erigido en modelo de referencia ${ }^{11}$. En primer lugar, porque su grado de proporcionalidad es considerado como uno de los más elevados del mundo, ya que ajusta casi de un modo exacto la relación entre votos y escaños. Y, en segundo lugar, porque consigue una importante personalización de la elección, al permitir que el elector pueda seleccionar la mitad de los candidatos que ocuparán los escaños del Bundestag, de manera que se logra una relación más directa entre el elector y el diputado. Además, tal y como está diseñado el sistema, se asegura que estén representadas diferentes tendencias ideológicas existentes en la sociedad sin que se produzca una excesiva fragmentación en la Cámara que pudieran dificultar la gobernabilidad $^{12}$.

Otro de los rasgos más característicos del sistema electoral alemán lo constituye el hecho de que en la configuración y en la aplicación del sistema electoral alemán ha tenido un especial protagonismo el Tribunal Constitucional federal, que, a lo largo de estos años, ha ido delimitando muy claramente las características del sistema, hasta el punto de obligar al legislador a reformar la Ley electoral para modificar algunos aspectos controvertidos que, de acuerdo con su jurisprudencia, eran contrarios a los principios básicos consagrados en la Ley Fundamental ${ }^{13}$.

En este sentido, el Tribunal Constitucional en las distintas ocasiones en la que ha tenido de juzgar el sistema electoral ha afirmado que el «sistema proporcional personalizado» es el que mejor se ajusta a la Constitución, ya que conserva todos los rasgos definitorios de los sistemas proporcionales con la única salvedad de que los electores tienen la capacidad de elegir a algunos de los diputados a través del Erststimme; y, de esta manera, se garantiza, por un lado, la igualdad electoral (Wablgeicheit) de los ciudadanos que participan en las elecciones, ya que con este sistema de cómputo de los votos y de distribución de los escaños se consigue que cada uno de los votos tenga el mismo valor en el resultado final, logrando, de esta manera, un reparto más exacto de los escaños y un resultado más justo, y por otro lado, también se garantiza la igualdad de oportunidades de los partidos políticos (Chancengleichbeit), puesto que todos los partidos que concurran a las elecciones obtendrán los escaños que correspondan a los votos recibidos de acuerdo con un criterio de proporcionalidad prácticamente exacto, reflejándose así todas las tendencias políticas que hay en la sociedad ${ }^{14}$. No obstante, más allá de avalar los rasgos básicos del sistema, ha rechazado algunos aspectos más cuestionables, como, por ejemplo, los mandatos adicionales o la regulación del voto electrónico.

11 Shugart, M.S., Wattenberg, M., Mixed-Member Electoral Systems: The Best of Both Worlds?, Oxford University Press, Oxford, 2001.

12 Fernández Esquer, C., «Los sistemas electorales de los Länder alemanes: panorámica general y tendencias de reforma», en Revista Española de Derecho Constitucional, 117, 2019, p. 104, 107 y 108.

13 Meyer, H., «Demokratische Wahl und Wahlsystem», op. cit., p. 540-541.

14 BVerfGE 95, 335 (352 y ss.). 


\section{REGULACIÓN DEL SISTEMA ELECTORAL}

En la Ley fundamental, como ya hemos indicado, no se regula el sistema electoral más allá de los principios que deben asegurarse para el ejercicio del derecho de sufragio activo y pasivo, a nivel nacional en el artículo 38.1 de la Ley Fundamental y para los Länder en el artículo 28 LF. En estos preceptos se establecen los principios que deben asegurarse para el ejercicio del derecho de sufragio activo y pasivo: universalidad (sufragio universal), libertad (sufragio libre), los principios del sufragio directo y secreto, e igualdad (sufragio igual).

Por ello, el sistema electoral alemán se rige, en primer lugar, por la Ley Electoral Federal (Bundeswablgesetz), de 7 de mayo de $1956^{15}$, en la versión actualizada de $1993^{16}$, que se encarga de regular las elecciones al Bundestag; en segundo lugar, por las Leyes electorales de cada uno de los Länder, que se ocupan de establecer las reglas para la elección de sus respectivos parlamentos,; en tercer lugar, por la Ley electoral federal para las elecciones al Parlamento Europeo (Europawablgesetz), de 8 de marzo de $1994^{17}$, y, finalmente, por otras normas jurídicas que conforman el derecho electoral germano, como el Reglamento Electoral Federal ${ }^{18}$ y la Ley de Control de las Elecciones ${ }^{19}$.

Además, también es de aplicación la Ley de partidos, ya que es esta norma la que establece el procedimiento a seguir para la selección de candidaturas, elemento fundamental del proceso electoral y que en Alemania tiene una regulación muy exhaustiva ${ }^{20}$.

\section{ELEMENTOS DEL SISTEMA ELECTORAL}

Los principales elementos que configuran el sistema electoral alemán son, en primer lugar, el sistema de doble voto; en segundo lugar, la determinación de las circunscripciones o distritos electorales; en tercer lugar, la presentación de

15 Bundeswahlgesetz (BWahlG) de 7 de mayo de 1956 (BGBl. I S., 383).). Esta Ley ha sufrido importantes modificaciones, siendo la última de 1 de julio de 2019 (BGBl. I, p. 834).

16 La Ley Electoral fue objeto de una reordenación, fruto de la cual se aprobó una nueva versión de la Ley en 1993. Bundeswablgesetz de 23 de julio de 1993 (BGBl. I S., p.1288).

17 Gesetz über die Wabl der Abgeordneten des Europäischen Parlaments aus der Bundesrepublik Deutschland (Europawablgesetz - EuWG), de 8 de marzo de 1994 (BGBl. I S. 423,)

18 Bundeswablordnung de 19 de abril de 2002 (BGBl. I, p. 1376). El Reglamento electoral federal regula la administración electoral, principalmente todo lo relacionado con los órganos electorales, así como cuestiones tales como la fijación de los resultados electorales, la convocatoria de nuevas elecciones, la repetición del proceso electoral, etc.

19 Wahlprïfungsgesetz de 12 de marzo de 1951 (BGBl. I, p. 166). Esta Ley regula la legitimación para poder interponer un recurso específico contra la elección en el plazo de un mes tras la proclamación de los resultados electorales.

20 Gesetz über die politischen Parteien (Parteiengesetz), de 31 de enero de 1994 (BGB1. I S. 149). 
candidaturas; en cuarto lugar, la determinación de barreras electorales; en quinto lugar, la fórmula electoral utilizada y, por último, el procedimiento de adjudicación de escaños.

\section{Sistema de doble voto}

Desde las elecciones de 1953 , cada elector dispone de dos votos ${ }^{21}$, aunque en realidad es una papeleta dividida en dos partes diferenciadas en las que figura, por un lado, el denominado primer voto (Ersttimme) en virtud del cual se elige de entre los candidatos individuales de los partidos que se han presentado en ese distrito uninominal, venciendo aquel que logre la mayoría de $\operatorname{votos}^{22}, y$, por otro lado, el segundo voto (Zweistimme) con el que se vota a una lista del partido en el Land correspondiente. Estas listas de partido son listas cerradas y bloqueadas.

El elector puede hacer efectivo los dos votos o bien ejercer el voto en uno de ellos dejando en blanco el otro o incluso puede votar al candidato de un partido con su primer voto y utilizar su segundo voto para votar a la lista de otro partido político. Eso es lo que se denomina el voto cruzado, voto dividido o voto estratégico (split vote o Stimmensplitting) ${ }^{23}$. Esta posibilidad no es demasiado frecuente - aproximadamente entre un 10 y un $20 \%$ en cada elección-, pero, en ocasiones, tiene una influencia determinante en el resultado electoral, y afecta, sobre todo, a los partidos más pequeños, que tienen menos opciones de lograr la victoria de sus candidatos de los distritos uninominales, y que suelen centrar sus campañas en conseguir los segundos votos y el mayor número de escaños posible ${ }^{24}$. Existen distintas razones que, desde la ciencia política, justifican el voto dividido ${ }^{25}$. Las más comunes son, por un lado, en el caso de electores de un partido minoritario que saben que el candidato de su partido en su distrito uninominal no tiene prácticamente opciones de obtener el escaño, por lo que vota al candidato de otro partido, y, por otro lado, en el caso de votantes de partidos mayoritarios que eligen al candidato de su partido con el primer

21 En las primeras elecciones tras la Segunda Guerra Mundial, de 1949, cada elector disponía de un único voto, pero desde la reforma de la ley electoral llevada a cabo en 1953 se optó por este sistema de doble voto que se ha mantenido hasta la actualidad.

22 No se exige una mayoría especial, por lo que basta con mayoría relativa para lograr el escaño.

23 Gschwend, T., «Ticket-splitting and strategic voting under mixed electoral rules: Evidence from Germany», en European Journal of Political Research, 46 (1), 2007, p. 1-23. NoHLEN, D., Sistemas electorales y partidos políticos, op. cit., p.265.

24 Turner, P. W., «Taktisch oder aufrichtig? Zur Untersuchung des Stimmensplittings bei Bundestagwahlen», en Zeitscrift für Parlamentsfragen,1, 1999, p. 163-165; PAPPI, F. U., TurNER, P. W., «Electoral Behavior in a Two-Vote System: Incentives for Tickets Splitting in German Bundestag Elections», en European Journal of Political Research, 41 (2) 2002, p. 207-232.

25 Schoen, H. «Stimmensplitting bei Bundestagswahlen: eine form taktischer Wahlentscheidung?», en Zeitscrift für Parlamentsfragen, 2, 1998, p. 223-244. 
voto y a la lista de otro partido que pudiera resultar clave para la formación de gobierno $^{26}$.

\section{Determinación de las circunscripciones o distritos electorales}

La división en distritos o circunscripciones electorales ha sido uno de los elementos que generaron más debate a la hora de diseñar el sistema electoral alemán, especialmente en el caso de los distritos uninominales en los que se emite en primer voto (Erststimmen). Tras la experiencia del sistema de Weimar organizado en torno a grandes circunscripciones y al voto despersonalizado que desincentiva la rendición de cuentas de los representantes políticos, se optó por un modelo en el que las circunscripciones electorales fueran relativamente pequeñas ${ }^{27}$.

No obstante, dadas las peculiares características del sistema alemán, el tamaño de la circunscripción no determina de manera esencial la valoración del voto. Ya hemos visto que se combinan elementos del sistema proporcional y del mayoritario, y que existen distritos uninominales para el primer voto, mientras que para el segundo voto la circunscripción electoral es el Land respectivo. Sin embargo, todos los votos emitidos tienen el mismo valor, ya que, para la distribución de escaños, se suman, a nivel federal, todos los votos obtenidos por un partido en los distintos Länder, creándose la ficción de que hubiera «listas a nivel federal». Esto es, para la distribución de escaños — que es lo importante- el distrito es único. De este modo, en la práctica, hay tres circunscripciones diferentes que se aplican en momentos distintos a la hora de emitir el voto y, sobre todo, a la hora de llevar a cabo el reparto de escaños ${ }^{28}$.

La primera circunscripción es la que se corresponde con los distritos uninominales. Existen 299 distritos uninominales en los que los candidatos compiten entre sí para obtener el escaño único que se disputa en cada uno de ellos. Los distritos electorales uninominales se establecen de manera proporcional a la población, sin que pueda haber una diferencia de población entre distritos superior al $15 \%{ }^{29}$. Es más, la ley dispone que, si se superase el $25 \%$, sería necesario llevar a cabo una remodelación de los distritos electorales. De esta manera, se asegura que todos los distritos uninominales tengan una población similar y no se afecte a la igualdad del $\operatorname{voto}^{30}$. Con anterioridad a la celebración de elecciones se procede a

26 Mestre-Aizpurua, F., «El sistema electoral alemán y su reciente reforma», en Cuadernos Manuel Jiménez Abad, nº 3, 2012, p. 82.

27 FERnÁndez Esquer, C., «Los sistemas electorales de los Länder alemanes: panorámica general y tendencias de reforma», op. cit., p 108.

28 Vázquez Lapuente, M. y Jiménez Seral, P., «El nuevo modelo de reparto de escaños en el sistema electoral alemán», op. cit., p. 112.

29 BWahlG, art. 3.

30 Mestre-Aizpurua, F., «El sistema electoral alemán y su reciente reforma», op. cit., p. 80. 
verificar si los distritos uninominales cumplen con lo establecido en la ley electoral y, si no es así, se procede a su adaptación. Y es en este ámbito en el que el Tribunal Constitucional ha llevado a cabo un control más estricto, con el fin de que no existan diferencias que pudieran afectar al principio de igualdad del voto $(\text { Wablgleichbeit })^{31}$.

La segunda circunscripción es el Land, y se aplica para el segundo voto. Cada uno de los dieciséis Länder constituye una circunscripción electoral en la que los partidos presentan sus candidaturas (Landeslisten). Además, y para poder determinar cuántos escaños le corresponden a cada Land, con carácter previo a la celebración de las elecciones, la Administración electoral reparte los 598 escaños iniciales del Bundestag entre los Länder en función de la población ${ }^{32}$.

Finalmente, la tercera circunscripción es todo el territorio nacional. Esta circunscripción es la que se utiliza a la hora de llevar a cabo el reparto de escaños. Todo el territorio actúa como una circunscripción única. De este modo, los partidos políticos van a competir por el reparto de los 598 escaños iniciales más aquellos otros escaños que se hayan añadido a lo largo del proceso electoral, ya sean en virtud de los llamados escaños adicionales o de los compensatorios ${ }^{33}$.

\section{Presentación de candidaturas}

\subsection{La presentación de firmas como requisito para las nuevas candidaturas (Unterschriftquoren)}

La Ley electoral federal fija los requisitos que han de cumplir las candidaturas electorales, entre los que destacan la exigencia de que los partidos que no hayan obtenido una representación parlamentaria de, al menos, cinco diputados, aporten una cantidad mínima de firmas (Unterschriftquoren) que avalen una candidatura, en concreto, 200 firmas de electores en el caso de los distritos uninominales y 2.000 firmas o el equivalente a un uno por mil de los electores de un Land, en el caso de listas en los Länder ${ }^{34}$.

A este respecto, el Tribunal Constitucional ha entendido que, a pesar de que el requisito de presentación de firmas podría considerarse una medida desfavorable para los partidos políticos de reciente creación, tal y como está diseñada, con un número de firmas y un porcentaje bastante reducido, no resulta contrario a la igualdad electoral ni tampoco a la igualdad de los partidos en la competición política,

31 BVerfGE 13, 123 (128 y ss.); 16, 30 (136).

32 Ipsen, J., Косн, T., «Wahlkreisgröße und Wahlrechtsgleichheit», en Niedersächsische Verwaltungsblätter - Zeitschrift für öffentliches Recht und öffentliche Verwaltung, 3, 1996, p. 269-274.

33 Fernández Esquer, C., «Los sistemas electorales de los Länder alemanes: panorámica general y tendencias de reforma», op. cit., p. 114.

$34 \mathrm{BWahlG}$, arts. 20.2 y 27.1. Los partidos que representan a las minorías nacionales quedan exentas de cumplir con esta exigencia. 
avalando la constitucionalidad de esta medida ${ }^{35}$. La doctrina también comparte la interpretación dada por el Tribunal Constitucional ${ }^{36}$, aunque algunos autores advierten que si el número de firmas fuera más elevado, esta medida sería más discutible desde la perspectiva constitucional, ya que se podría anticipar la decisión de los ciudadanos, conculcando así el secreto del sufragio garantizado en el artículo $38 \mathrm{LF}^{37}$.

\subsection{El proceso de selección de candidatos y de elaboración de las listas electorales}

Uno de los aspectos más característicos y mejor valorados del sistema electoral alemán es que debido al sistema de doble voto se permite una mayor participación ciudadana, ya que al elector se le permite elegir a un candidato concreto de entre lo que se presentan en su distrito electoral y, a la vez, elegir a una lista de candidatos pertenecientes a un partido político. De este modo, se logra, por un lado, una vinculación más directa entre los electores y el diputado elegido en el distrito uninominal, y, por otro, que los electores se sientan partícipes de la composición del Parlamento mediante el voto al partido de su preferencia ${ }^{38}$. Y es por ello por lo que se presta especial atención al modo en el que se lleva a cabo el proceso interno de selección de los candidatos en el seno de los partidos políticos, ya que, como en el sistema alemán las candidaturas son listas cerradas y bloqueadas, sin posibilidad de que el elector pueda cambiar el orden de los candidatos, se considera imprescindible que dicho proceso de selección de los candidatos sea resultado de un proceso democrático que debe cumplir con una serie de reglas fijadas en la ley, del mismo modo que ocurre con otros momentos del proceso electoral ${ }^{39}$.

Tanto la ley de partidos como la ley electoral establecen que el proceso de selección de los candidatos que se presentan a las elecciones parlamentarias, ya sea a nivel nacional (Bundestag), regional (Landtag) o local, debe realizarse mediante voto secreto, siguiendo las reglas establecidas en estas leyes y, de acuerdo con lo previsto en los propios estatutos de las formaciones políticas ${ }^{40}$, de acuerdo con una serie de estándares democráticos que han de cumplirse en los diferentes momentos de la elaboración de las candidaturas, en especial los principios de universalidad, libertad e igualdad reconocidos en el art. 38 de la Constitución ${ }^{41}$.

35 BVerFGE 3, 19 (27), y 30, 227 (246).

36 Badura, P., Staatsrecht, $3^{\text {a }}$ ed., op. cit., p. 447-448; Jülich, H.-C., Chancengleichheit der Parteien, Duncker \& Humblot, Berlín, 1967, p. 115; LEE, H.-T. Chancengleichbeit der politischen Parteien, op. cit., p. 43.

37 Stern, K., Das Staatsrecht der Bundesrepublik Deutschland, op. cit., p. 309.

38 Vázquez Lapuente, M., Jiménez Seral, P., «El nuevo modelo de reparto de escaños en el sistema electoral alemán», op. cit., p. 108.

39 BVerfGE 89, 243. Maurer, H., Staatsrecht I, Beck, Munich, 2a ed, 2001, p. 363.

40 ParteiG, art. 17. Morlok, M., «Demokratie und Wahlen», en P. Badura/ H. Dreier, Festschrift 50 Jahre Bundesverfassungsgericht, II Vol., Mohr Siebeck, Tübingen, 2001, pp. 558-608; IPSEN, J., «Artikel 17», en Ipsen, J., Parteiengesetz Kommentar, C.h. Beck, Munich, $2^{\mathrm{a}}$ ed., 2018.

41 Morlok, M., Merten. H., Parteienrecht, Mohr Siebeck, Tübingen, 2018, p. 221. 
La participación en este tipo de procesos selectivos está restringida a los afiliados del partido y le corresponde a la normativa interna de cada organización política definir quién puede ser considerado afiliado, así como las condiciones que se deben reunir para participar en los $\operatorname{mismos}^{42}$. En todo caso, solo podrán participar quienes tengan reconocido el derecho de sufragio activo, es decir, no podrán participar en estos procesos de selección de candidatos ni menores de edad ni tampoco extranjeros que puedan estar registrados en el partido, a pesar de que puedan formar parte del mismo ${ }^{43}$.

En lo que respecta al proceso de nominación de los candidatos al Bundestag, la ley exige a los partidos seleccionar a sus candidatos al Parlamento nacional bien por el voto directo y secreto de todos los afiliados en cada circunscripción o bien mediante la celebración de una asamblea o convención celebrada en el distrito electoral correspondiente, en el que los afiliados puedan participar directa o indirectamente a través de sus delegados y establece diferentes sistemas de elección de candidatos, distinguiendo entre los candidatos uninominales y los candidatos que forman parte de una lista electoral ${ }^{44}$.

Para la selección de los candidatos uninominales es precisa la participación de los afiliados de la circunscripción electoral correspondiente ya sea de manera directa, a través de procesos electivos tipo primarias o indirectamente, a través de compromisarios o delegados. Lo más habitual es que la selección se lleve a cabo en una asamblea de delegados. La votación tiene carácter secreto y se requiere mayoría absoluta en la primera vuelta y mayoría simple en segunda votación. No obstante, la ley confiere al comité ejecutivo regional del partido la posibilidad de intervenir en el proceso de selección. En este sentido, se establece que el órgano de dirección del partido a nivel regional, en caso de discrepancia con los resultados de la votación efectuada por la asamblea de delegados, podría oponerse a la selección de un candidato, esto es, ejercer el derecho de veto, y obligar a repetir la votación. En ese caso, el resultado de la segunda votación realizada por la asamblea de delegados es la decisión final y tiene carácter vinculante ${ }^{45}$. Sólo se puede ser candidato por una única circunscripción.

En cuanto al procedimiento para la elaboración de las candidaturas de los partidos, los candidatos que figuran en la lista electoral son seleccionados por las asambleas de delegados de las agrupaciones locales del partido en la circunscripción en la que se presenta la candidatura, o bien por las asambleas regionales del partido. La ley electoral deja los detalles relativos a la selección de dichos delegados a los estatutos de los partidos, que, a su vez, se suelen remitir a los estatutos

42 BWahlG, art. 21. IPSEN, J., «Artikel 21», op cit., p. 869 y 871.

43 SChÖnberger, S., «The impact of the German Party Regulation on the elections and the creation of the new Federal Government», en Federalismi.it, Rivista di Diritto Pubblico italiano, comparato, europeo, 24, 2018 , p.5.

44 BWahlG, art. 18 y ss.

45 BWahlG, art. 21. 
propios y a las normas de organización de las agrupaciones regionales, limitándose, en la mayoría de los casos, a trazar los aspectos básicos del procedimiento de selección ${ }^{46}$. En todo caso, debe asegurarse la capacidad de los afiliados para presentar propuestas y para participar de manera democrática en la elección, así como la posibilidad de que los candidatos se presenten debidamente y puedan presentar sus programas ${ }^{47}$.

En la asamblea de delegados se vota cada puesto en la lista, en orden descendente, desde el primero hasta el último de la lista, debiendo obtener mayoría absoluta cada uno de ellos. Por regla general, los puestos de la lista electoral que tienen más probabilidades de salir elegidos y que se corresponden con los primeros puestos en la lista suelen ser propuestos por el Comité ejecutivo, aunque esta propuesta se somete también a votación y la asamblea tiene la facultad de modificar dicha propuesta si la mayoría de los delegados así lo decide. Asimismo, cualquiera de los afiliados tiene la facultad de presentar propuestas. Por otro lado, los últimos puestos de la lista electoral pueden ser votados de manera conjunta, si así lo estima la asamblea. Cabe destacar también que, a diferencia de lo que ocurre con los candidatos uninominales, no se prevé que el comité ejecutivo regional o nacional pueda ejercer ningún tipo de veto a la decisión de la asamblea.

Como ya hemos indicado, el proceso de selección de los candidatos electorales constituye un aspecto especialmente relevante en el sistema político y electoral alemán, hasta el punto de que es objeto de control tanto por la administración electoral como por los tribunales ${ }^{48}$. En este sentido, los partidos políticos han de remitir a las autoridades electorales competentes un documento en el que consten los candidatos que van a formar parte de sus candidaturas, tanto en los distritos uninominales como en las listas, y en que ha de especificarse dónde y cuándo se ha celebrado la asamblea para la elección de dichos candidatos, el número de miembros presentes, así como el resultado de las votaciones llevadas a cabo en ese proceso de selección, de manera que la administración electoral pueda comprobar que el partido ha cumplido con las exigencias legales y si no es así, se rechazará la candidatura. Del mismo modo, se permite que cualquier elector que considere que el procedimiento de elaboración de las candidaturas no se ajusta a derecho puede ponerlo en conocimiento del Bundestag e incluso acudir a los tribunales ${ }^{49}$. Así ha ocurrido, por ejemplo, en el proceso de selección de los candidatos del partido AFD (Alternative für Deutschland) en la región de Sarre en 2017, que, tras

46 BWahlG, arts. 26 y 27. Son los estatutos de los partidos los que se encargan de detallar cómo se eligen a los delegados que forman parte de la asamblea de delegados encargados de la selección de los candidatos, la convocatoria, el quorum necesario, etc.

47 Morlok, M., Merten. H., Parteienrecht, op. cit., p. 222.

48 Mager, U., «Die Kontrolle der innerparteilichen Kandidatenaufstellung im Wahlprüfrungsverfahren», DÖV 1995, p. 9 y ss.

49 Schönberger, S., «The impact of the German Party Regulation on the elections and the creation of the new Federal Government», op. cit., p. 6. 
denuncias presentadas por varios afiliados ante tribunales civiles se ordenó la repetición del proceso de selección ${ }^{50}$.

Por su parte, y en cuanto al control jurisdiccional, cabe destacar la actuación del Tribunal Constitucional de Hamburgo que anuló las elecciones celebradas el 2 de junio de 1991 para elegir al Parlamento de esa región, al entender que el procedimiento de elaboración de la candidatura del partido cristiano demócrata CDU no había respetado ni los principios ni el procedimiento que se establece en la ley para la elaboración de las candidaturas, al lesionar los derechos de los afiliados a proponer candidatos para su nominación y obligó, por ello, a la repetición de las elecciones en ese territorio ${ }^{51}$.

\subsection{Las cuotas de género}

Otro de los elementos a tener en cuenta a la hora de analizar la presentación de candidaturas es el establecimiento de medidas de acción positiva, mediante cuotas de género, para lograr la igualdad entre mujeres y hombres en la representación política y garantizar así la efectiva integración de las mujeres en los cargos electivos de decisión de los partidos políticos y del Estado.

Pues bien, a pesar de las recomendaciones efectuadas por distintos organismos internacionales y regionales de protección de los derechos humanos y de la Unión Europea ${ }^{52}$ y aunque en Alemania existen distintas leyes en las que se exige el cumplimiento de estas cuotas en distintos sectores, como, por ejemplo, en los consejos de administración de las grandes empresas, no existe ninguna norma federal que prevea la existencia de cuotas en las candidaturas, ya que se ha venido entendiendo que el establecimiento de cuotas podría suponer una injerencia en la libertad de elección y en el principio de libertad organizativa de los partidos políticos consagrada constitucionalmente ${ }^{53}$. La única excepción es la reciente aproba-

50 Oberlandesgericht Saarbücken n. 1 U 80/17, de 12 de julio de 2017.

51 HambVerfG 3/93, de 4 de mayo (DVB1. 93, 1071 y ss.). En la doctrina, vid. KoeING, C., ¿ «Schadenserstatzanspruche nach verfassungsgerichtlicher Ungultigkeitserklärung von Parlamentswahlen?» en Die Offentliche Verwaltung, vol. 7, 1994, pp. 286 y ss.; IPSEN, J., «Kandidatenaufstellung, innerparteiliche Demokratie und Wahlprufungsrecht. Anmerkung zum Urteil des Hamburgischen Verfassungsgerichts von 4. Mai 1993. HverfG 3/93», en Zeitschrift fur Parlamentsfragen, Vol. 2, 1994, pp. 235 y ss.; MAGER, U., «Die Kontrolle der innerparteilichen Kandidataufstellung im Wahlprufungsverfahren», en Die Offentliche Verwaltung, vol. 1, 1995, pp. 7 y ss.;

52 Entre otras, destacan la Convención de Naciones Unidas sobre la eliminación de todas las formas de discriminación contra la mujer (CEDAW), de 1979, la Recomendación del Consejo de Ministros del Consejo de Europa de 2003 sobre participación equilibrada de mujeres y hombres en la toma de decisiones políticas y públicas y el Acuerdo estratégico de la Unión Europea para la igualdad de género 2016-2019, que instan a los Estados a lograr que la participación de mujeres y hombres en los órganos de toma de decisiones no sea inferior al $40 \%$.

53 Ipsen, J., «Artikel 17», op. cit., p. 123; PenZ, M., «Frauenquoten innerhalb politischer Parteien», en $D O ̈ V, 2015$, p. 963 y ss. 
ción en 2019 de dos leyes de paridad (Paritätgesetze) en el Land de Brandenburgo y en el Land de Turingia, que establecen la obligación de que en las listas de candidatos al Parlamento regional haya el mismo número de hombres y de mujeres ${ }^{54}$.

No obstante, y aunque no exista una ley que exija la paridad o una participación equilibrada entre géneros, algunos partidos políticos han incorporado cuotas voluntarias de género en sus estatutos y normas internas para garantizar que un determinado número de candidatas figuren en sus listas electorales ${ }^{55}$. Así, hasta ahora, los partidos políticos que han incorporado las cuotas de género han sido el partido socialdemócrata SPD, que desde 1990 ha dispuesto que al menos el $40 \%$ de las listas electorales estén integradas por mujeres, el Partido de Socialismo Democrático (PDS), la Izquierda (Die Linke) y los Verdes, que han establecido «listas cremallera» encabezadas por mujeres. Por su parte, la CDU/CSU no ha fijado cuotas de género en sus normas internas, aunque a través de una directriz no vinculante se establecía la necesidad de «tener en cuenta a las mujeres» y se comprometió, de facto, a que las mujeres ocupasen el segundo puesto en las listas electorales y que hubiera «cuotas flexibles» en virtud de las cuales las candidaturas incorporasen al menos a tres mujeres en cada bloque de diez candidatos. Por último, otros partidos como el partido liberal FDP o Alternativa para Alemania (AFD) no poseen cuotas de género y se han manifestado abiertamente en contra de éstas ${ }^{56}$.

Aun así, hay que resaltar que a pesar de que algunos partidos tienen fijadas cuotas y que en la actualidad una mujer ocupa la cancillería y la presidencia del principal partido de la oposición y de otros partidos con representación parlamentaria, no se ha logrado la paridad. De hecho, el porcentaje de mujeres que forman parte del Bundestag se sitúa en el 30,7\%, el número más bajo en los últimos veinte años.

\section{Las barreras electorales}

Las barreras electorales o Sperrklauseln, son una condición que han de cumplir los partidos políticos para acceder al reparto de escaños (ya sean del Bundestag o de cualquiera de los Landtag). A tenor de lo que establece la Ley Electoral

54 La Paritägesetz del Land de Brandenburgo se aprobó el 31 de enero de 2919 y entrará en vigor en verano de 2020 y la Paritätgesetz del Land de Turingia se aprobó el 5 de julio de 2019. Ambas leyes han sido fuertemente criticadas, han sido recurridas ante el Tribunal Constitucional y está pendiente de que se dicte sentencia.

55 EBSEn, I., Quotenregelung für Frauen und Männer in Parteistatuten, 1998; LANGE, K., «Frauenquoten im politichen Parteien», en NJW, 1988, p. 2201 y ss.

56 PARLAMENTO EUROPEO. DIRECTORATE GENERAL INTERNAL POLICIES OF THE UNON, Sistemas electorales de cuotas de género y su aplicación en Europa, Septiembre de 2008, disponible en https:// www.europarl.europa.eu/thinktank/es/document.html? reference=IPOL-FEMM_ET\%282008\%29408309 
Federal, existen dos tipos de barreras electorales: por un lado, la llamada barrera porcentual, y, por otro, la condición de haber ganado en un mínimo de circunscripciones uninominales. Sin embargo, no es necesario que los partidos cumplan con ambos requisitos, pues son alternativos, y cumpliendo cualquiera de los dos se accede al reparto de escaños ${ }^{57}$.

La primera de las barreras electorales, la barrera porcentual (die Prozentklause), consiste en la exigencia de haber obtenido como mínimo un $5 \%$ de los votos (segundos votos o Zweitstimme) emitidos a nivel nacional. La segunda barrera (die Grundmandatklausel) consiste en la exigencia de haber obtenido tres mandatos directos, es decir, haber logrado la mayoría de los votos en, al menos, tres distritos uninominales (primeros votos o Erststimme $)^{58}$. Hay que señalar, además, que estas barreras (haber obtenido un $5 \%$ de votos o tres mandatos directos en los distritos uninominales), no se aplican a los partidos que representan a las minorías nacionales $^{59}$, aunque esta posibilidad, hasta ahora, no se ha producido nunca.

Alemania fue uno de los primeros países en exigir que los partidos alcanzasen un porcentaje mínimo de votos para poder acceder a los Parlamentos, dificultando, así, la proliferación de partidos en el Parlamento ${ }^{60}$. Con el establecimiento de estas barreras se intenta evitar que se repita la experiencia del sistema de partidos en la época de Weimar y, en consecuencia, se admite que quiebre la igualdad en sentido estricto de los partidos con el objetivo de garantizar la existencia de un sistema en el que primen los partidos consolidados con posibilidades de formar mayorías estables ${ }^{61}$. En este sentido, hay que indicar que, en las primeras elecciones celebradas tras la Segunda Guerra Mundial, las elecciones de 1949, la barrera porcentual se aplicó a nivel del Land y no a nivel nacional, lo que provocó que algunos partidos políticos con una fuerte influencia en un solo territorio consiguieran acceder al Bundestag, que contó en esa legislatura con once formaciones políticas diferentes y no se lograse el objetivo de frenar la «atomización» parlamentaria. Por ello, a partir de ese momento se modificó la Ley electoral fijando que para que las formaciones políticas puedan acceder al reparto de escaños deben lograr, al menos, el $5 \%$ de los votos en el conjunto del territorio nacional ${ }^{62}$.

57 Pointner, M., Bundeswablrecht Kommentar, Verlag für Verwaltungspraxis Rehm, Munich, 1980, p. 26; Hegels, W., «Chancengleichheit der Parteien», en ZRP, 9, 1969, p. 105; H.-T. LEE, Chancengleichbeit der politischen Parteien, op. cit., p. 29.

58 Wild, M., Die Gleichbeit der Wabl, op. cit., pp. 222 a 236.

59 BwahlG, art. 6.6. Las minorías reconocidas son la danesa, la frisona y la sorbia. Las minorías danesa y frisona están situadas en el norte de Alemania, mientras que la minoría sorbia se sitúa en la región de Lusacia, en la frontera germano-polaca.

60 Fernández Esquer, C., «Los sistemas electorales de los Länder alemanes: panorámica general y tendencias de reforma», op. cit., p. 120.

61 Stern, K., Das Staatsrecht der Bundesrepublik Deutschland, op. cit., pp. 311-312; WENner, U., Sperrklauseln im Wablrecht der Bundesrepublik Deutschland, Lang, Bonn, 1986, p. 88.

62 Nohlen, D., «El sistema electoral alemán y el Tribunal Constitucional Federal. La igualdad electoral a debate», en Ferrer Mac-Gregor E., Zaldívar Lelo de Larrea (Coords.), La ciencia del derecho 
Del mismo modo, y en relación con la barrera de los mandatos directos, en un primer momento bastaba con haber logrado un mandato directo en un distrito uninominal, pero, por los mismos motivos, se elevó la barrera a los tres mandatos directos, lo que provoca que, en la práctica, sea casi imposible poder acceder al Parlamento por esta segunda vía, ya que exige que un partido regional tenga una gran fuerza electoral en un determinado territorio, que le permita compensar la falta de votos en el resto del territorio nacional. Hasta ahora, solo ha ocurrido con el PDS, el partido sucesor del partido comunista de la Alemania oriental, que ha conseguido obtener representación en el Bundestag por esta vía ${ }^{63}$.

El Tribunal Constitucional alemán ha tenido oportunidad de pronunciarse sobre la constitucionalidad de fijar por ley barreras electorales para poder obtener representación parlamentaria y, de acuerdo con una jurisprudencia constante del alto Tribunal ${ }^{64}$, las barreras contenidas en las leyes electorales, tanto de la Federación como de los Länder, se ajustan a la Constitución ${ }^{65}$. El Tribunal, en una interpretación funcionalista, considera que hay que evitar una excesiva fragmentación del sistema de partidos que podría entorpecer e, incluso, paralizar el normal funcionamiento del Parlamento y, con ello, obstaculizar la formación de gobiernos estables ${ }^{66}$, y que para conseguir ese objetivo el establecimiento de Sperrklauseln resulta justificado y proporcionado, a pesar de que esta decisión pueda afectar a la igualdad de oportunidades de los partidos políticos ${ }^{67}$. Aun así, es preciso señalar aquí que el Tribunal Constitucional hizo una excepción a su interpretación consolidada respecto de la fijación de barreras electorales, con ocasión de las primeras elecciones tras la reunificación de Alemania de 1990. En estas elecciones se decidió suspender la aplicación de estas barreras con el objetivo de permitir que los partidos de la antigua República Democrática Alemana tuviesen la oportunidad de lograr representación parlamentaria. Pues bien, el Tribunal avaló la suspensión de la aplicación de las barreras electorales por ser una situación excepcional y meramente coyuntural. Además, en el caso de las elecciones al Parlamento Europeo, el Tribunal Constitucional ha declarado que las barreras electorales para ese proceso electoral en concreto sí que lesiona el principio de igualdad de sufragio y la igualdad de oportunidades de los partidos políticos, ya que, por las propias características de la elección, no hay ninguna causa que justifique el establecimiento de estas barreras $^{68}$.

procesal Constitucional. Estudios en Homenaje a Héctor Fix-Zamudio en sus cincuenta años como investigador del Derecho, Vol. VI, UNAM, México, 2008, p. 791.

63 Meyer, H., «Wahlgrundsätze, Wahlverfahren, Wahlprüfung», en IsEnSEe / KIRCHHHOF (Eds.), Handbuch des Staats Rechts, vol. III, $3^{\text {a }}$ ed, C. F. MülLER, Heidelberg, 2005, p. 569-570.

64 BVerfGE 82, 322, de 29 de septiembre de 1990.

65 BVerfGE 1, 208; 4, 31; 4, 375; 5, 77; 6, 84; 6, 99; 6, 104; 6, 121; 47, 253; 48, 64; 67, 65 .

66 BVerfGE 1, 208 (249); 6, 84 (92 y ss); 13, 243 (247). Magiera, S., «Art. 38», en SACHS, M. Grundgesetz Kommentar, $8^{\mathrm{a}}$ ed, 2018, C. H. BECK, Munich, p. 1245.

67 BVerfGE 24, 300 (341).

68 BVErfGE 82, 322, de 29 de septiembre de 1990. 
Por su parte, la mayor parte de la doctrina considera adecuada la existencia de las Sperrklauseln, aunque también hay autores que no encuentran suficiente justificación constitucional a las mismas. Entre estos últimos se afirma que las barreras electorales no juegan un papel decisivo en la garantía del funcionamiento del Parlamento; así, se entiende que las diferencias que pueden surgir entre incluir a todos los partidos que han obtenido votos en el reparto de escaños o excluir a los que han conseguido un número mínimo de votos no son relevantes, y que la repercusión que tiene para el buen funcionamiento del Bundestag es prácticamente inexistente ${ }^{69}$. Incluso algunos autores van más allá y consideran que no existe ninguna causa que justifique la creación de estas barreras, ya que con ellas se estaría vulnerando el pluralismo político, la igualdad electoral, consagrada en el art. $38 \mathrm{LF}$ y la igualdad de oportunidades de los partidos políticos reconocida y garantizada en el art. $21 \mathrm{LF}$, al dificultar la aparición de nuevas formaciones a nivel nacional ${ }^{70}$.

\section{Fórmula electoral}

La fórmula electoral, es decir, el cálculo matemático que se utiliza en todo sistema proporcional para distribuir los escaños en función de los votos, se ha modificado en varias ocasiones en el sistema alemán, siempre con el objetivo de lograr una mayor proporcionalidad, de manera que haya una correspondencia lo más fidedigna posible entre los votos y los escaños y, además, para evitar ciertos efectos anómalos que pudieran distorsionar este objetivo. Así, a lo largo de su reciente historia democrática, para asignar los escaños correspondientes a los segundos votos, esto es, a las Landeslisten $^{71}$, se ha utilizado, primero, el método D’Hondt desde 1949 hasta 1985, después, la fórmula Hare/Niemeyer, hasta 2008 y, finalmente, la fórmula Sainte-Laguë/Schepers desde las elecciones de $2009^{72}$.

69 De los autores que forman parte de este sector crítico, destacan, WENNER, U., Sperrklauseln im Wablrecht der Bundesrepublik Deutschland, op. cit., p. 226 y ss.; Preuss, U.K., «Artikel 21, Abs. 1, 3», en E. DenNINGER, Kommentar zum Grundgesetz für die Bundesrepublik Deutschland. Reibe Alternativ-Kommentar, 1984, Rdn. 48; o Lowestein, en «Das Schicksal der Wahlreform», en ZfP, 1970, p. 25. En términos semejantes se expresa Forsthoff, E., Der Staat der Industriegesellschaft, 1970, pp. 89-93; Jesse, E., Wablrecht zwischen Kontinuität und Reform, 1985, p. 234 y ss.; y WILD, M., Die Gleichbeit der Wabl, op. cit., pp. 227 a 228.

70 LEE, H.-T., Chancengleichbeit der politischen Parteien, op. cit., p. 40; JüLICH, H.-C., Chancengleichbeit der Parteien, op. cit., p. 112 y ss.; PETER, C., Wablabsprachen politischer Parteien und ibre rechtlicher Grenzen, 1964, p. 111. Bosch, A., Guía del sistema electoral, Universidad Autónoma de Barcelona, Bellaterra, 2007, p. 110.

71 Hay que recordar que los primeros votos (Erststimmen) correspondientes a los distritos uninominales se rigen por el sistema de mayoría, de manera que el candidato que haya obtenido el mayor número de votos en el distrito electoral es el que logra el escaño. No es preciso lograr mayoría absoluta ni obtener una determinada diferencia de sufragios respecto del segundo candidato más votado ni se celebra una segunda vuelta.

72 . Meyer, H., «Wahlgrundsätze, Wahlverfahren, Wahlprüfung», op. cit., p. 578-579. 
El método D`Hondt consiste en dividir a través de distintos divisores la cantidad total de votos obtenidos por los partidos que han concurrido a las elecciones, de manera que se producen secuencias con los cocientes ordenados de forma decreciente para cada partido, y asignando los escaños a los promedios más altos. Los restos se asignan al partido que haya obtenido más votos totales, de manera que tiende a favorecer a los partidos políticos mayoritarios, especialmente en el caso de circunscripciones de tamaño reducido.

En 1983 se decidió sustituir el sistema D’Hondt por la fórmula Hare/Niemeyer. La fórmula Hare/Niemeyer, también conocida como la «fórmula del resto más elevado», consiste en multiplicar el total de votos válidos obtenidos por cada partido por el número total de votos válidos emitidos. Los partidos obtienen tantos representantes como números enteros resulten y los puestos restantes se distribuyen según el valor del número decimal posterior. Las razones que motivaron este cambio fueron jurídicas y políticas. Así, por un lado, se argumentó que la fórmula Hare/Niemeyer respeta más la proporcionalidad, ya que transforma de manera más exacta los votos en escaños sin favorecer especialmente a los partidos mayoritarios y permite, por tanto, más posibilidades de que los partidos pequeños puedan lograr representación ${ }^{73}$. En este sentido, hay que resaltar que el Tribunal Constitucional bávaro declaró pocos años después, en 1992, la inconstitucionalidad del sistema D’Hondt, al considerar que favorecía a los grandes partidos cuando se aplicaba a circunscripciones de magnitud reducida, de forma que el porcentaje de votos obtenido por un partido no era proporcional al porcentaje de escaños que se les adjudicaba, afectando a la igualdad electoral y a la igualdad de oportunidades de los partidos políticos ${ }^{74}$. Además, por otro lado, esta reforma tuvo también un fuerte condicionante político, ya se produjo como consecuencia de los acuerdos de Gobierno de coalición entre la CSU/CDU y el FDP, que era el partido que podría resultar beneficiado por el cambio en la fórmula electoral ${ }^{75}$.

Por su parte, la fórmula Sainte-Laguë/Schepers, también conocida como método del divisor con redondeo estándar, es una fórmula a través de la cual se utiliza el promedio mayor para asignar los escaños. En concreto, se van realizando divisiones a través de distintos divisores de los totales de los votos obtenidos por cada uno de los partidos, produciéndose una secuencia de cocientes decrecientes de cada partido. Los escaños se asignarían a los cocientes de promedio más alto ${ }^{76}$.

73 SAn Segundo, J. M., «Posibles aportaciones de los sistemas electorales de Estados de estructura compuesta a nuestro modelo electoral, en Asamblea: Revista Parlamentaria de la Asamblea de Madrid, 30, 2014, p. 302. G. Leibholz / H.J. Rinck / D. Hesselberger, Grundgesetz, op. cit., Art. 38, Rdn. 236; H.-T. LEE, Chancengleichheit der politischen Parteien, op. cit., pp. 27-28; R. SCHMIDT, Staatsorganisationsrecht, op. cit., p. 37.

74 Gavara, J. C., Vallés, F., Los regímenes electorales territoriales en los Estados compuestos. Alemania, Estados Unidos e Italia, op. cit., p. 110.

75 Scarrow, S., Germany: the Mixed-Member System as a Political Compromise, en Shugart, S., Wattenberg, M. P. (eds.), Mixed Member Systems: the Best of Both Worlds?, op. cit., p. 66.

76 Norris, P., Electoral Engineering: Voting, Rules and Political Behavior, Cambridge University Press, 2004, p. 51. Colomer, J., The Handbook of Electoral System Choice, Palgrave Macmillan, 2004, p. 553. 
El motivo del cambio a esta fórmula, en sustitución a la utilizada hasta ese momento, la Hare/Niemeyer, es porque se ha comprobado que este método es uno de los que consiguen una mayor proporcionalidad ${ }^{77}$.

A través de este método, tal y como dispone la ley electoral, se van efectuando diferentes aproximaciones para lograr el divisor adecuado. Así, en primer lugar, se divide el número total de votos por el número de escaños. Se obtiene así un primer divisor. En segundo lugar, se dividen los votos obtenidos por cada partido por ese primer divisor. Los números resultantes se redondean al número entero más cercano de la forma estándar, esto es, las cantidades cuya parte decimal sea superior a 0.5 se redondea hacia el número superior, las que tengan decimales inferiores a 0.5 se redondea hacia el número inferior y las que sean igual a 0.5 podrían redondearse hacia arriba o hacia abajo respetando el número total de escaños a distribuir ${ }^{78}$. Si después de llevar a cabo estas operaciones, la suma de todos los escaños coincide con el número de escaños a repartir, se asignarían los escaños a cada partido político. Sin embargo, si no se produce tal coincidencia, habrá que repetir el paso anterior, pero con un divisor mayor o menor, según corresponda, hasta que la suma de los cocientes, una vez efectuado el redondeo, coincida con el de escaños a repartir ${ }^{79}$.

\section{Procedimiento de adjudicación de escaños}

Una vez celebrada la votación se inicia el procedimiento de reparto de escaños, al que sólo podrán acceder aquellas formaciones políticas que hayan superado las barreras electorales correspondientes. Este procedimiento de adjudicación de escaños es estrictamente proporcional ${ }^{80}$. Como hemos visto, el Bundestag está integrado, inicialmente, por 598 diputados, de los cuales 299 se adjudican a los candidatos elegidos a través de distritos electorales uninominales y los otros 299 restantes a los candidatos que integran las listas electorales a nivel de cada $\mathrm{Land}^{81}$. El número de escaños puede variar, incrementándose el tamaño de la Cámara siempre que sea preciso. De hecho, en cada convocatoria electoral el número final de escaños en el Bundestag ha ido variando en función de los resultados obtenidos por los partidos políticos una vez que se lleva a cabo el proceso de adjudicación de escaños. Así, por ejemplo, en la legislatura actual, tras las elecciones de 2017, el Bundestag ha alcanzado los 709 escaños. Y es, precisamente, la manera en la que

77 Benoit, K, "Which Electoral Formula is the Most Proportional? A New Look with New Evidence», en Political Analysis 8 (4), 2000, pp. 381-388.

78 BWahlG, art. 6.2.

79 VázQuez Lapuente, M., Jiménez Seral, P., «El nuevo modelo de reparto de escaños en el sistema electoral alemán», op. cit., p. 110-111.

80 C. Vidal Prado, El sistema electoral alemán y su posible implantación en España, op. cit., p. 51.

81 BWahlG, art. 1. 
se regulan estos escaños adicionales lo que ha provocado una gran controversia en Alemania en los últimos años, que ha sido resuelta por el Tribunal Constitucional al declarar en 2008 su inconstitucionalidad y que trajo como consecuencia la reforma de la Ley electoral federal estableciendo un nuevo sistema de reparto de escaños y de regulación de los escaños adicionales.

\subsection{Procedimiento de adjudicación de escaños antes de la reforma de 2013}

Antes de la reforma de 2013, el procedimiento de adjudicación de escaños se realizaba en dos fases diferenciadas. En la primera fase se llevaba a cabo el recuento de votos que había obtenido cada partido con el objetivo de determinar cuántos escaños en el Bundestag les iban a corresponder. Para ello, se sumaban todos los segundos votos que había obtenido cada uno de los partidos políticos en cada Land (Zweitstimmen), es decir, los votos a las listas, y se conseguía, de esta manera, el número total de votos que había obtenido cada partido a nivel nacional. A ese número resultante se le aplicaba la fórmula electoral Hare-Niemeyer, y de ahí resultaba el número de escaños que le correspondía a cada partido a nivel nacional. Una vez que se conocía el número total de escaños que había obtenido cada partido político, se llevaba a cabo un nuevo reparto (con la misma fórmula electoral) para saber cuántos de esos escaños le correspondía al partido en cada uno de los Länder ${ }^{82}$.

En la segunda fase, los partidos políticos procedían a la adjudicación de los escaños que habían obtenido. En este sentido, primero se asignaba escaño a los candidatos que habían sido elegidos a través del primer voto (Erststimme), es decir, los elegidos directamente en los distritos electorales uninominales (mandatos directos). Y, a continuación, el resto de los escaños se asignaba a los candidatos que aparecían en la lista electoral del Land elegidos a través del segundo voto (Zweitstimme), hasta completar el número de escaños que hubiera obtenido cada partido $^{83}$.

El sistema, tal y como estaba diseñado, producía algunos efectos anómalos y controvertidos $^{84}$. El primero de ellos, el conocido como escaños adicionales, suple-

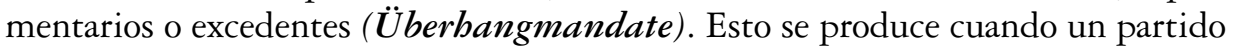
consigue en una circunscripción más escaños en los distritos uninominales que el número de escaños que le correspondería en esa circunscripción en función del

82 Hesse, K., Grundzüge der Verfassungsrechts der Bundesrepublik Deutschland, op. cit., p. 254; BADURA,

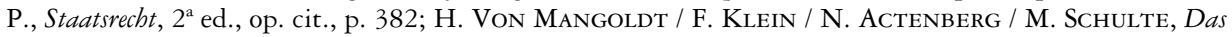
Bonner Grundgesetz, 1991, Art. 38, Rdn. 161.

83 Maurer, H., Staatsrecht, op. cit., p. 404; Nohlen, D., «Tendencias recientes en desarrollo de los sistemas electorales en el mundo», en Sistema de gobierno, sistema electoral y sistema de partidos políticos, 1999, pp. 117-118.

84 Behnke, J., «The Strange Phenomenon of Surplus Seats in the German Electoral System», en German Politics, 16 (4), p. 496-517. 
reparto proporcional ${ }^{85}$. En esos casos, si el número de escaños conseguido por un partido en la circunscripción con los primeros votos fuera superior a la cantidad que le correspondería a la lista del Land por los segundos votos, el partido conservaría estos escaños adicionales, incrementándose así el número total de diputados en el Bundestag. Los escaños adicionales pueden producirse porque se haya producido voto cruzado (split vote), esto es, que los electores hayan votado a partidos diferentes en cada uno de sus votos o bien porque el partido que ha logrado más escaños en los distritos uninominales (mandatos directos) lo haya obtenido con poco margen de diferencia respecto del siguiente partido político ${ }^{86}$.

Esta situación de los escaños adicionales surge como consecuencia de la interrelación de las reglas de la mayoría y de la proporcionalidad y podría haberse evitado si el legislador hubiera fijado un sistema de reparto de escaños en virtud del cual únicamente se hubieran tenido en cuenta para el reparto proporcional los 299 escaños correspondientes a los segundos votos, esto es, a los votos de las listas. Sin embargo, al establecerse que, para efectuar el cálculo de cuántos escaños le corresponde a cada formación política se utiliza la cantidad total de escaños del Bundestag, incluidos los que se van a atribuir a los candidatos elegidos en distritos uninominales y si a eso se suma el hecho de que, en todo caso, hay que garantizar los escaños a los diputados elegidos con los primeros votos, pues de lo contrario se estaría perdiendo la propia esencia del sistema personalizado de la elección, da como resultado la necesidad de tener que crear esos escaños adicionales para adaptar el sistema a los resultados obtenidos ${ }^{87}$.

Resulta llamativo, cuando menos, que aunque los escaños adicionales han existido desde siempre, esta situación no hubiera suscitado ningún debate ni ninguna controversia hasta mediados de los años 90, cuando el número de escaños adicionales se incrementó notablemente y fueron determinantes para la formación de gobierno, ya que el $75 \%$ de estos escaños fueron a parar a la CDU, lo que le permitió lograr una mayoría suficiente para poder formar gobierno de coalición con los liberales. Sin embargo, esta «anomalía» se consideraba aceptable, siempre que el número de escaños adicionales fuera pequeño ${ }^{88}$. Así lo entendió incluso el Tribunal Constitucional que tuvo la oportunidad de enjuiciar la constitucionalidad de este tipo de escaños adicionales en 2007 y avaló su existencia, al interpretar que era una medida adecuada para garantizar, por un lado, la proporcionalidad del sistema y, por otro, la personalización del voto, siempre que el número de escaños adicionales fuera reducido ${ }^{89}$.

85 Meyer, H., «Wahlgrundsätze, Wahlverfahren, Wahlprüfung», op. cit., p. 570 y ss.

86 Nohlen, D., Sistemas electorales y partidos políticos, op. cit., p. 257 y 271.

87 Gavara, J. C., Vallés, F., Los regímenes electorales territoriales en los Estados compuestos. Alemania, Estados Unidos e Italia, Congreso de los Diputados, Madrid, 2007, p. 123.

88 Vázquez Lapuente, M., Jiménez Seral, P., «El nuevo modelo de reparto de escaños en el sistema electoral alemán», op. cit., p. 110.

89 BVerfGE 95, 335, de 27 de abril de 1997. 
Otro de los aspectos más controvertidos del sistema es el denominado peso negativo del voto (Negative Stimmgewichte). El peso negativo del voto o valor del voto inverso se produce como consecuencia del reparto de escaños adicionales. Consiste en que el aumento de segundos votos de lista puede provocar una pérdida de escaños de la candidatura o que una pérdida en votos de lista suponga un aumento de los escaños de un partido, esto es que, que un partido que con más segundos votos hubiera obtenido menos escaños y viceversa, que partidos con menos votos lograran más escaños ${ }^{90}$.

Esta situación anómala se hizo evidente en la opinión pública en las elecciones de 2005. En esas elecciones falleció un candidato electoral, por lo que las autoridades decidieron retrasar la elección de ese distrito electoral unos días, de manera que se pudieran confeccionar las papeletas electorales con el nuevo candidato. Pues bien, durante esos días que transcurrieron entre la elección general y la elección parcial en ese territorio — el distrito de Dresden — se sucedieron las informaciones en los medios de comunicación sobre qué efectos podrían tener los resultados de esa circunscripción en cuanto a la adjudicación de escaños, llegando a la paradoja de que como la CDU ya había obtenido un escaño adicional correspondiente a los primeros votos, aunque este partido obtuviera pocos votos en los segundos votos tendría asegurados los escaños. Esta situación provocó un debate muy intenso en la ciudadanía y en los medios de comunicación y, como consecuencia de ello, varios partidos políticos presentaron un recurso ante el Tribunal Constitucional, que determinó en 2008 que este efecto, el peso negativo del voto, era inconstitucional, al afectar al principio de igualdad de voto y al principio de representación proporcional y obligó al legislador a establecer una regulación alternativa compatible con la Constitución ${ }^{91}$. Esta interpretación la mantuvo el Tribunal Constitucional, de nuevo en $2012^{92}$. Por ello, y tras varios intentos fallidos, y después de un intenso debate político y jurídico, se aprobó la reforma de la Ley electoral en 2013, con un nuevo sistema de adjudicación de escaños ${ }^{93}$.

\subsection{Procedimiento actual de adjudicación de escaños}

Tras la reforma de 2013 además de los escaños adicionales se crearon los escaños compensatorios (Ausgleichsmandaten) que se asignan a los partidos políticos con el objetivo de mantener la proporcionalidad entre todos ellos y evitar el efecto del peso negativo del voto ${ }^{94}$.

90 Magiera, S., «Art. 38», op. cit., p. 1246; Vidal Prado, C., El sistema electoral alemán y su posible implantación en España, Tirant lo Blanch, Valencia, 2012, p. 44 y 45.

91 BVerfGE 121, 266, de 3 de julio de 2008.

92 BVerfGE, NVwZ 2012, 1102, de 25 de julio de 2012.

93 Morlok, M., Merten. H., Parteienrecht, Mohr Siebeck, Tübingen, 2018, p. 217.

94 FERnÁNDez Esquer, C., «Los sistemas electorales de los Länder alemanes: panorámica general y tendencias de reforma», op. cit., p. 113. 
El procedimiento de asignación de escaños se lleva a cabo de la siguiente manera. Con carácter previo a la adjudicación, los 598 escaños que componen inicialmente el Bundestag se reparten entre los Länder en función de la población y a continuación se distinguen dos fases diferenciadas: una primera fase en la que se determina cuántos escaños en el Bundestag van a corresponder a cada partido político y una segunda fase en la que los partidos políticos proceden a la adjudicación de los escaños que les han correspondido entre sus candidatos.

La primera fase, en virtud de la cual se precisa cuántos escaños ha obtenido cada una de las formaciones políticas es la que ha sufrido más cambios tras la reforma. En este sentido, para poder determinar cuántos escaños corresponden a cada partido hay que efectuar una serie de operaciones consecutivas.

Así, en primer lugar, se hace el reparto de los escaños correspondientes a los distritos uninominales. Así, teniendo en cuenta los primeros votos (Erstimmen), obtiene el escaño aquel candidato más votado en cada uno de 299 distritos. Estos escaños ya adjudicados se agrupan por partido y por el Land al que pertenezca el distrito correspondiente.

En segundo lugar, se procede al recuento de los segundos votos (Zweistimmen), esto es, de los votos de las Landenlisten obtenidos por los partidos, sumando para cada uno de ellos los conseguidos en todos los Länder. Una vez que se tienen los resultados de los segundos votos de todos los partidos a nivel nacional, se eliminan a todos los que no hayan superado las barreras electorales y se calcula el porcentaje que le corresponde a cada partido político sobre el total votos. Aplicando la fórmula electoral Saint-Lägue/Schepers se hace un primer reparto provisional de los escaños que obtendría cada partido en cada uno de los Länder ${ }^{95}$.

En tercer lugar, se recopilan los datos correspondientes a los escaños obtenidos por los partidos gracias a los primeros votos y a los segundos votos. $\mathrm{Y}$, en aquellos casos en los que el partido haya logrado más escaños en los distritos uninominales que los que les correspondería por listas, se añadirán esos escaños adicionales en los Länder que los precisen ${ }^{96}$. Del mismo modo, y con el objetivo de garantizar que la distribución global de escaños en todo el país sea proporcional y que no se vea alterado por la incorporación de los escaños adicionales se deberán incrementar el número de escaños mediante los escaños compensatorios. Se añadirán todos los escaños compensatorios que sean precisos para reflejar la proporcionalidad exacta y para evitar el efecto negativo del voto. Para ello, se utilizará de nuevo la fórmula electoral. Hay que indicar que la cifra de escaños adicionales y compensatorios no es idéntica, es decir, no hay que añadir un escaño compensatorio por cada escaño adicional, sino que en función de los escaños adicionales habrá que fijar los escaños compensatorios que sean necesarios para restaurar la proporcionalidad. Así, por ejemplo, en los últimos comicios de 2017 hubo 46 escaños adicionales, 36 de los 
cuales correspondieron al CSU, y se establecieron 75 escaños compensatorios, alcanzando la cifra total de 111 escaños más que tuvieron que añadirse a los 589 escaños que, inicialmente, componen el Bundestag ${ }^{97}$.

Como consecuencia del incremento de escaños adicionales y compensatorios se vuelve a realizar una nueva asignación de escaños a los partidos a nivel fede$\mathrm{ral}^{98}$, repartiéndose entre las listas regionales de ese partido proporcionalmente a los votos obtenidos en sus listas, garantizándose, en todo caso, que hay suficientes escaños para los obtenidos por los primeros votos.

La segunda fase consiste en la adjudicación de los escaños por parte de los partidos políticos. Así, una vez que se ha producido el reparto definitivo de escaños, tras la incorporación de los escaños adicionales y los complementarios, si los hubiera, cada partido procede a repartir los escaños entre sus candidatos. La asignación se realiza en cada Land y comienza atribuyendo escaño a los candidatos que hayan sido elegidos directamente mediante los primeros votos y, a continuación, el resto de los escaños se asignan a los candidatos que forman parte de la lista del partido en ese Land, hasta completar el número de escaños que le hayan correspondido ${ }^{99}$.

A diferencia de lo que ocurría en el anterior sistema de adjudicación de escaños, ahora se prevé una adjudicación después de haber realizado dos repartos: primero, se asignan de manera provisional los escaños de cada Land entre las listas de los partidos y se incluyen los escaños adicionales. Y, en el segundo reparto, se asignan los escaños a nivel nacional pero respetando el mínimo fijado en el primer reparto provisional y añadiendo los escaños compensatorios que sean precisos para asegurar la proporcionalidad global del sistema. Después, los partidos distribuyen los escaños obtenidos entre sus listas y sus mandatos directos, pero sin añadir más escaños a los que ya se les han asignado ${ }^{100}$.

Este nuevo sistema de adjudicación de escaños no ha estado exento de críticas. Así, y a pesar de haber logrado evitar el efecto del peso negativo del voto y que se mantenga la proporcionalidad de una manera más exacta, se alerta sobre las dimensiones que está adoptando el Bundestag, con 631 escaños en 2013 y 709 en 2017, lo que afecta, sin duda, al propio funcionamiento de la Cámara y a otros muchos aspectos, como, por ejemplo, a la financiación de los partidos ${ }^{101}$. Además, se afirma que el nuevo sistema es tan complejo que contraviene el principio de transparencia, al resultar prácticamente imposible para los ciudadanos comprender cómo es el proceso de adjudicación de escaños, afectando, por ello, a su propia legitimidad ${ }^{102}$.

97 Fernández Esquer, C., «Los sistemas electorales de los Länder alemanes: panorámica general y tendencias de reforma», op. cit., p. 114.

98 BWahlG, art. 6.6.

99 Morlok, M., Merten. H., Parteienrecht, op. cit., p. 219-220.

100 Vázquez Lapuente, M., Jiménez Seral, P., «El nuevo modelo de reparto de escaños en el sistema electoral alemán», op. cit., p. 113-117.

101 Morlok, M., Merten. H., Parteienrecht, op. cit., p. 220.

102 Dehmel, N., Jesse, E., «Das neue Wahlgesetz zur Bundestagswahl 2013. Eine Reform der Reform der Reform ist unvermeidlich», en Zeitschrift für Parlamentsfrgen, 44, 2013. 


\section{A MODO DE CONCLUSIÓN}

Como consideración final podíamos afirmar que el sistema electoral alemán sigue manteniendo, a pesar de los diferentes cambios de mayor o menor calado que se han ido incorporando a lo largo de los años, las notas características que le ha hecho ser considerado un modelo ejemplar. Así, por un lado, la proporcionalidad del sistema sigue siendo muy elevada, aunque para lograrlo se haya tenido que incrementar el tamaño del Bundestag. Por otro lado, la participación se sigue asegurando, no sólo a los electores, a través de los primeros votos, sino también en el seno de los partidos políticos mediante una regulación muy detallada de los mecanismos de selección de los candidatos conforme a los principios democráticos. Y, finalmente, la gobernabilidad, otro de los aspectos que suelen ser objeto de preocupación en sistemas proporcionales multipartidistas también ha quedado garantizada, generalmente, mediante acuerdos de coalición y con la presencia de diferentes fuerzas políticas que representan las distintas corrientes ideológicas de la sociedad, pero sin que se fragmente en exceso el espectro parlamentario. Ahora bien, el hecho de que el sistema alemán pueda ser un modelo de referencia, eso no significa que pueda ser extrapolable a España. Ambos sistemas, aun siendo proporcionales, tienen enormes diferencias de concepción y de aplicación. Aun así, sin duda, el modelo alemán nos puede aportar ideas para la reflexión y el debate.

$$
* * *
$$

TiTLE: The German electoral system under review.

ABSTRACT: This paper analyzes the German electoral system, which is characterized by being a reference model in the world, combining elements of both majority and proportional systems and in which the candidates' nomination within political parties is especially relevant. In addition, there is an analysis of the latest legal reforms that affected the seat allocation procedure and the influence of the Federal Constitutional Court in the delimitation of the whole system.

RESUMEN: El trabajo que aquí presentamos es un análisis del sistema electoral alemán, que se caracteriza por ser un modelo de referencia en el mundo, al combinar elementos del sistema mayoritario y proporcional y en el que se presta especial atención al procedimiento de elaboración de candidaturas en el seno de los partidos políticos. Además, se analizan las últimas reformas efectuadas en el procedimiento de asignación de escaños y la labor desempeñada por el Tribunal Constitucional federal en la delimitación del sistema.

KEY WORDS: candidate nomination, electoral system, Constitutional Court.

Palabras Clave: selección de candidatos electorales, sistema electoral, Tribunal Constitucional.

FECHA DE RECEPCIÓN: 15.12.2019

FECHA DE ACEPTACIÓN: 19.02.2020 
